\title{
Facteurs associés à l'existence d'une politique maison sans fumée dans les ménages québécois
}

\author{
É. Ouedraogo, M.D. (1); F. Turcotte, M.D. (2); M.J. Ashley, M.D., D.H.P. (3); J.M. Brewster, Ph.D. (4); R. Ferrence, Ph.D. (5)
}

\section{Résumé}

Le domicile est une importante source d'exposition à la fumée de tabac environnante pour les non-fumeurs, dont les enfants, lorsqu'ils cohabitent avec des fumeurs. Nous tentons d'identifier les facteurs sociodémographiques associés à l'existence d'une politique maison sans fumée (PMSF) dans les ménages québécois «fumeurs ». Les associations sont comparées à 3 autres provinces du Canada (Ontario, Colombie-Britannique, NouvelleÉcosse). Il s'agit d’une étude transversale portant sur 2648 répondants. Des analyses de régression logistique sont utilisées. Peu de ménages québécois "fumeurs " (21\%) ont une PMSF; la présence d'un adulte non-fumeur est fortement liée à l'existence d'une PMSF; celle d'un enfant de moins de 6 ans l'est peu comparativement aux autres provinces et celle d'un adolescent ne l'est point. Outre le bénéfice de la PMSF sur la santé de l'enfant, peut-être faut-il mettre de l'avant celui sur son comportement tabagique ultérieur. Une implication pour la promotion de la santé pourrait être une campagne dirigée vers les non-fumeurs cohabitant avec des fumeurs, incitant à moins de tolérance.

Mots clés : fumée de tabac environnante, maison sans fumée, facteurs

sociodémographiques, ménages québécois

\section{Introduction}

La nocivité de la fumée de tabac environnante (FTE) pour la santé des nonfumeurs est maintenant bien démontrée ${ }^{1-3}$. $\mathrm{Au}$ Canada, des mesures restreignant l'exposition à la FTE sont en place au travail et dans les lieux publics. ${ }^{i}$ Plusieurs études montrent que ces mesures ont pour conséquences, outre l'amélioration de la santé et du confort des non-fumeurs, la réduction de la consommation tabagique des fumeurs ${ }^{4}$.

Les lieux privés demeurent toutefois une importante source d'exposition à la FTE notamment pour les enfants d'âge préscolaire. L'accueil favorable de la restriction tabagique dans les lieux publics ou de travail et la baisse de l'acceptabilité sociale du tabagisme font penser que cette mesure pourrait être étendue aux lieux privés. Ainsi, en Ontario, la proportion de nonfumeurs favorables à l'interdiction totale de fumer en présence de jeunes enfants a augmenté de 15,4\% entre 1992 et 1996 tandis que celle de fumeurs opposés à telle restriction a diminué de $8,6 \%$ au cours de la même période 5 .

Les données sur l'existence d'une politique maison sans fumée (PMSF) des ménages canadiens deviennent disponibles mais restent peu abondantes. En 1995, selon une étude sur la PMSF menée par Santé
Canada auprès des familles comptant des enfants âgés de 12 ans et moins, 19\% de ces familles avaient une PMSF complète, $44 \%$ avaient une PMSF partielle et $37 \%$ n'en avaient pas ${ }^{6-7}$. Le taux de réponse de cette étude était cependant de $50 \%$.

En dépit d'une baisse remarquable de la prévalence du tabagisme, le Québec reste au-dessus de la moyenne canadienne en matière d'exposition à la FTE. Selon la dernière Enquête de surveillance de l'usage du tabac au Canada (ESUTC) conduite en 2006, la prévalence des enfants âgés de 0-17 ans exposés à la FTE à domicile est de $21.6 \%$ comparativement à $11.2 \%$ pour l'ensemble du Canada ${ }^{8}$.

$\mathrm{Au}$ meilleur de nos connaissances, il n'existe pas de données populationnelles décrivant l'effet de facteurs sociodémographiques sur la présence d'une PMSF au Québec. L’objectif de cette étude est d'identifier les caractéristiques sociodémographiques des ménages et des répondants décrivant le mieux l'existence d'une PMSF dans les ménages québécois comptant au moins un fumeur et de les comparer à celles de 3 autres provinces du Canada (Ontario, Colombie-Britannique et Nouvelle-Écosse).

\section{Méthodologie}

Il s'agit d'une étude transversale basée sur la population. Les données proviennent de l'Enquête nationale sur l'exposition à

i Des mesures restreignant l'exposition à la FTE sont en place même dans le lieu « privé » de la voiture : en Ontario, une nouvelle loi en vigueur depuis le $\mathbf{2 1}$ janvier $\mathbf{2 0 0 9}$ permet de sanctionner ceux qui seraient trouvés en train de fumer à bord d'un véhicule dans lequel il y aurait des enfants âgés de moins de seize ans.

Coordonnées des auteurs

1 Département Médecine Sociale et Préventive, Faculté de médecine, Université Laval (Québec)

2 Département Médecine Sociale et Préventive, Faculté de médecine, Université Laval (Québec)

3 Dalla Lana School of Public Health, University of Toronto (Ontario)

4 Ontario Tobacco Research Unit, Dalla Lana School of Public Health, University of Toronto (Ontario)

5 Ontario Tobacco Research Unit, Centre for Addiction and Mental Health University of Toronto (Ontario)

Correspondance : Éva Ouedraogo, M.D., Département Médecine Sociale et Préventive, Faculté de médecine, Université Laval, eva.ouedraogo@inspq.qc.ca 
la FTE dans les environnements privés du Canada; elles ont été colligées par l'Institut de Recherche Sociale (York University) sous la direction de l'Unité de Recherche sur le Tabac de l'Ontario (OTRU) de juin 2001 à janvier 2002.

L'échantillonnage et les questionnaires ont déjà été décrits ${ }^{9}$. Brièvement, l'échantillon initial compte 14600 ménages dont les répondants ont tous rempli un premier questionnaire visant à établir le statut tabagique du ménage et à déterminer la présence d'enfants de moins de 18 ans. Afin d'obtenir des informations relatives à l'existence d'une PMSF, un second échantillon de ménages "fumeurs " a été sélectionné. Cet échantillon surreprésente les ménages comprenant à la fois au moins un adulte fumeur et un enfant dans le but de fournir une taille suffisante pour les analyses statistiques; selon ces critères, 5000 ménages provenant de toutes les provinces canadiennes ont été sélectionnés. Parmi eux, aux fins de cette étude, 2648 ménages fumeurs avec ou sans enfants répartis dans 4 provinces (Québec, Ontario, Colombie-Britannique et NouvelleÉcosse) ont été retenus. Bien que l'enquête s'étende à tout le Canada, le choix de la Colombie-Britannique et de l'Ontario tient de la pertinence à comparer, d'une part, le Québec avec des provinces qui affichent, selon la littérature, de meilleures prévalences en termes de PMSF et, d'autre part, avec la Nouvelle-Écosse, qui est comparable au Québec sauf en ce qui a trait au contexte culturel.

Un ménage fumeur est défini comme un ménage dans lequel au moins une personne de 18 ans et plus fume quotidiennement ou occasionnellement des cigarettes, cigares, cigarillos ou pipe. Bien que des informations aient été colligées sur toutes les personnes "fumeurs » du ménage, peu de personnes de moins de 18 ans vivent loin de leurs parents et sont à même d'établir leurs propres règles à domicile? .

Les participants ont été sélectionnés à partir de numéros de téléphone générés par ordinateur. Les informations ont été obtenues à l'aide d'un questionnaire administré lors d'une interview téléphonique assistée par ordinateur selon la méthode
CATI (Computer Assisted Telephone Interview). Pour assurer un taux de réponse optimal, un nombre maximal de 14 appels est effectué pour tout numéro de téléphone ne permettant pas d'établir un contact lors d'un premier appel, dont 10 pendant la soirée et la fin de semaine.

La variable dépendante est la PMSF. Une maison sans fumée est un domicile à l'intérieur duquel tous les occupants s'abstiennent, en tout temps, de fumer. Le comportement des visiteurs et des invités en relation avec la politique d'abstention de tabac n'est pas considéré. La PMSF est mesurée à partir de la réponse à la question suivante : "Fumez-vous des cigarettes, des cigares ou des cigarillos ou la pipe dans la maison tous les jours, de temps en temps ou pas du tout? ». Dans le cas où il y a plus d'un fumeur dans la maison, la question est posée au répondant pour chacun des autres fumeurs. Ces informations permettent de définir une variable à 2 niveaux de réponse : mesures restrictives totales vis-à-vis de la fumée de tabac à domicile d'une part, et mesures restrictives partielles ou aucune mesure restrictive d'autre part.

Les résultats sont exprimés par rapport de prévalences pondérées. L'instauration d'un système de pondération répond à la nécessité de tenir compte de l'inégale probabilité de sélection d'un ménage selon sa composition puisque les ménages fumeurs avec enfants avaient une plus grande probabilité d'être sélectionnés que ceux sans fumeur ni enfant. Et aussi pour tenir compte d'une inégale répartition des ménages selon la province d'origine. Un coefficient de pondération a ainsi été attribué à chaque type de ménage.

Une analyse univariée de régression logistique non conditionnelle a d'abord permis d'identifier les variables les plus fortement associées à la PMSF. Ensuite, une analyse multivariée a été effectuée. Les variations provinciales de l'effet d'une caractéristique sociodémographique sur la présence de la PMSF ont été évaluées à partir d'un modèle de régression logistique stratifié pour la province et ajusté pour les autres caractéristiques. Pour éviter un problème de colinéarité, les variables apportant la même information n'ont pas été incluses simultanément dans le modèle. Les rapports de prévalences pondérées obtenus permettent d'estimer l'association entre chaque variable et l'existence d'une PMSF avec un intervalle de confiance de $95 \%$.

Les analyses statistiques ont été effectuées avec le logiciel SAS.

\section{Résultats}

\section{Caractéristiques sociodémographiques et prévalences de la PMSF}

Un total de 2648 répondants représentant des ménages avec au moins un adulte fumeur a accepté d'être interrogé dans les 4 provinces. Le taux de réponse à l'enquête est de $62 \%$. Les tableaux 1 et 2 présentent respectivement les caractéristiques des répondants et des ménages. Les répondants sont un peu plus souvent du sexe féminin que masculin; plus de la moitié vit en couple. Près de $50 \%$ d'entre eux a un niveau de scolarité inférieur ou égal à l'école secondaire. Soixante-trois pour cent sont des fumeurs de cigarettes et/ou de pipe, cigares ou cigarillos dont $80 \%$ fument quotidiennement. Un peu plus du tiers des ménages vit avec au moins un enfant, qui dans $37 \%$ des cas a moins de 6 ans. Au Québec, 949 ménages fumeurs ont accepté de répondre au questionnaire. Les caractéristiques de l'échantillon québécois sont comparables à celles de l'échantillon total. Compte tenu du grand nombre de valeurs manquantes sur le revenu familial, l'effet de cette variable n'a pas été pris en compte dans les analyses d'association.

La prévalence pondérée de la PMSF au Québec est de $21 \%$; elle est significativement différente de celles de l'Ontario et de la Colombie-Britannique, respectivement de $43.7 \%$ ( $p<0.0001$ ) et de $52.1 \%$ $(p<0.0001)$. Elle n'est pas différente statistiquement de celle observée en Nouvelle-Écosse (32\%), $p=0.64$.

\section{Facteurs associés à la présence d'une PMSF au Québec}

Le tableau 3 présente les résultats des analyses uni et multivariées. Plusieurs variables caractérisant le répondant sont significativement associées à la PMSF en analyse univariée mais ne le sont plus lorsque l'on tient compte des autres 
TABLEAU 1

Caractéristiques des répondants dans les provinces du Québec, de l’Ontario, de la Colombie-Britannique et de la Nouvelle-Écosse

\begin{tabular}{|c|c|c|c|c|c|c|c|c|c|c|c|c|c|c|c|}
\hline \multirow[t]{2}{*}{ Variables } & \multicolumn{3}{|c|}{ Québec } & \multicolumn{3}{|c|}{ Ontario } & \multicolumn{3}{|c|}{ Colombie-Britannique } & \multicolumn{3}{|c|}{ Nouvelle-Écosse } & \multicolumn{3}{|c|}{ Totala $^{a}$} \\
\hline & $n^{b}$ & $\% c$ & $\% P^{d}$ & $\mathbf{n}$ & $\%$ & $\% \mathbf{P}$ & $\mathbf{n}$ & $\%$ & $\% \mathbf{P}$ & $\mathbf{n}$ & $\%$ & $\% \mathbf{P}$ & $\mathbf{n}$ & $\%$ & $\% P$ \\
\hline \multicolumn{16}{|c|}{ Groupe d'âge (en années) } \\
\hline $18-24$ & 156 & 16,5 & 22,0 & 165 & 14,0 & 19,2 & 52 & 14,7 & 19,2 & 18 & 13,8 & 19,1 & 391 & 15,0 & 20,1 \\
\hline $25-64$ & 718 & 76,1 & 70,5 & 949 & 80,8 & 75,1 & 267 & 75,6 & 71,2 & 103 & 79,2 & 73,5 & 2037 & 78,3 & 72,9 \\
\hline$\geq 65$ & 69 & 7,3 & 7,5 & 61 & 5,2 & 5,7 & 34 & 9,6 & 9,6 & 9 & 6,9 & 7,4 & 173 & 6,6 & 7,0 \\
\hline Total & 943 & & & 1175 & & & 353 & & & 130 & & & 2601 & & \\
\hline \multicolumn{16}{|l|}{ Sexe } \\
\hline Féminin & 549 & 57,8 & 56,0 & 669 & 55,5 & 52,8 & 194 & 53,7 & 49,2 & 66 & 50,0 & 42,9 & 1478 & 55,8 & 52,9 \\
\hline Masculin & 400 & 42,1 & 44,0 & 537 & 44,5 & 47,2 & 167 & 46,3 & 50,8 & 66 & 50,0 & 57,1 & 1170 & 44,2 & 47,1 \\
\hline Total & 949 & & & 1206 & & & 361 & & & 132 & & & 2648 & & \\
\hline \multicolumn{16}{|l|}{ Statut tabagique } \\
\hline Fumeur actuel & 646 & 68,4 & 63,5 & 814 & 68,0 & 62,9 & 222 & 61,7 & 57,0 & 97 & 74,0 & 71,8 & 1779 & 67,5 & 62,6 \\
\hline Ancien fumeur & 137 & 14,5 & 15,8 & 157 & 13,1 & 15,1 & 59 & 16,4 & 47,0 & 16 & 12,2 & 12,2 & 369 & 14,0 & 15,5 \\
\hline Non-fumeur & 162 & 17,1 & 20,7 & 227 & 18,9 & 22,0 & 79 & 21,9 & 26,0 & 18 & 13,8 & 16,0 & 486 & 18,5 & 21,9 \\
\hline Total & 945 & & & 1198 & & & 360 & & & 131 & & & 2634 & & \\
\hline \multicolumn{16}{|l|}{ État civil } \\
\hline Conjoint & 560 & 59,4 & 58,9 & 695 & 58,1 & 57,0 & 205 & 57,1 & 56,5 & 84 & 63,6 & 63,6 & 1544 & 58,7 & 57,8 \\
\hline $\begin{array}{l}\text { Célibataire/ } \\
\text { séparé/divorcé }\end{array}$ & 383 & 40,7 & 41,1 & 502 & 41,9 & 43,0 & 154 & 42,9 & 43,5 & 48 & 36,4 & 36,4 & 1087 & 41,3 & 42,2 \\
\hline Total & 943 & & & 1197 & & & 359 & & & 132 & & & 2631 & & \\
\hline \multicolumn{16}{|l|}{ Niveau de scolarité } \\
\hline $\begin{array}{l}\text { Baccalauréat } \\
\text { ou plus }\end{array}$ & 132 & 14,0 & 13,3 & 218 & 18,4 & 18,6 & 70 & 19,5 & 20,5 & 15 & 11,6 & 14,5 & 435 & 16,6 & 17,0 \\
\hline Postsecondaire & 318 & 33,8 & 35,6 & 564 & 47,6 & 47,1 & 186 & 51,8 & 51,1 & 25 & 19,4 & 18,9 & 1093 & 32,4 & 33,1 \\
\hline $\begin{array}{l}\text { Secondaire } \\
\text { ou moins }\end{array}$ & 491 & 52,2 & 51,1 & 402 & 34,0 & 34,3 & 103 & 28,7 & 28,4 & 89 & 69,0 & 66,6 & 1085 & 51,0 & 49,9 \\
\hline Total & 941 & & & 1184 & & & 359 & & & 129 & & & 2613 & & \\
\hline \multicolumn{16}{|c|}{ Perception de la FTE comme : } \\
\hline $\begin{array}{l}\text { Problème de santé } \\
\text { très important }\end{array}$ & 566 & 59,9 & 59,8 & 844 & 70,4 & 70,0 & 257 & 71,4 & 71,4 & 96 & 73,3 & 74,2 & 1763 & 67,9 & 67,1 \\
\hline $\begin{array}{l}\text { Problème de } \\
\text { santé peu ou } \\
\text { pas important }\end{array}$ & 380 & 40,1 & 40,2 & 354 & 29,6 & 30,0 & 103 & 28,6 & 28,6 & 35 & 27,7 & 25,8 & 872 & 33,1 & 32,9 \\
\hline Total & 946 & & & 1198 & & & 360 & & & 131 & & & 2635 & & \\
\hline \multicolumn{16}{|l|}{ FTE au lieu d'emploi } \\
\hline Restriction totale & 370 & 70,9 & 70,3 & 472 & 78,3 & 78,9 & 143 & 90,5 & 89,6 & 31 & 68,9 & 66,0 & 1016 & 76,8 & 77,3 \\
\hline $\begin{array}{l}\text { Restriction } \\
\text { partielle }\end{array}$ & 131 & 29,1 & 29,7 & 131 & 21,7 & 21,1 & 15 & 9,5 & 10,4 & 14 & 31,1 & 34,0 & 291 & 23,2 & 22,7 \\
\hline Total & 501 & & & 603 & & & 158 & & & 45 & & & 1307 & & \\
\hline \multicolumn{16}{|c|}{ Le total peut différer de la taille de l'échantillon (2648) du fait de valeurs manquantes ou inappropriées. } \\
\hline $\mathrm{n}$ représente la taille $\mathrm{r}$ & de l'é & intillon & & & & & & & & & & & & & \\
\hline$\%$ représente les pro| & is no & déré & & & & & & & & & & & & & \\
\hline
\end{tabular}


TABLEAU 2

Caractéristiques des ménages dans les provinces du Québec, de l’Ontario, de la Colombie-Britannique et de la Nouvelle-Écosse

\begin{tabular}{|c|c|c|c|c|c|c|c|c|c|c|c|c|c|c|c|}
\hline \multirow[t]{2}{*}{ Variables } & \multicolumn{3}{|c|}{ Québec } & \multicolumn{3}{|c|}{ Ontario } & \multicolumn{3}{|c|}{ Colombie-Britannique } & \multicolumn{3}{|c|}{ Nouvelle-Écosse } & \multicolumn{3}{|c|}{ Total $^{a}$} \\
\hline & $n^{b}$ & $\%{ }^{c}$ & $\% \mathbf{P}^{\mathbf{d}}$ & $\mathbf{n}$ & $\%$ & $\% \mathbf{P}$ & $\mathbf{n}$ & $\%$ & $\% \mathbf{P}$ & $\mathbf{n}$ & $\%$ & $\% \mathbf{P}$ & n & $\%$ & $\% \mathbf{P}$ \\
\hline \multicolumn{16}{|c|}{ Présence de $\geq 1$ adulte non-fumeur } \\
\hline Oui & 576 & 60,7 & 68,9 & 719 & 59,6 & 68,8 & 225 & 62,3 & 70,0 & 72 & 54,5 & 61,7 & 1592 & 60,1 & 68,7 \\
\hline Non & 373 & 39,3 & 31,1 & 487 & 40,4 & 31,2 & 136 & 37,7 & 30,0 & 60 & 45,5 & 38,3 & 1056 & 39,9 & 31,3 \\
\hline Total & 949 & & & 1206 & & & 361 & & & 132 & & & 2648 & & \\
\hline \multicolumn{16}{|l|}{ Présence d'enfant } \\
\hline Oui & 449 & 47,3 & 35,5 & 621 & 51,5 & 39,8 & 171 & 47,4 & 35,2 & 64 & 48,5 & 37,7 & 1305 & 49,3 & 37,7 \\
\hline Non & 500 & 52,7 & 64,5 & 585 & 48,5 & 60,2 & 190 & 52,6 & 64,8 & 68 & 51,5 & 62,3 & 1343 & 50,7 & 62,3 \\
\hline Total & 949 & & & 1206 & & & 361 & & & 132 & & & 2648 & & \\
\hline \multicolumn{16}{|l|}{ Âge des enfants } \\
\hline 5 ans et moins & 155 & 34,6 & 33,3 & 236 & 38,5 & 38,1 & 69 & 41,1 & 41,0 & 20 & 31,7 & 32,3 & 480 & 37,2 & 36,8 \\
\hline $6-17$ ans & 293 & 65,4 & 66,7 & 377 & 61,5 & 61,9 & 99 & 58,9 & 59,0 & 43 & 68,3 & 67,7 & 812 & 62,8 & 63,2 \\
\hline Total & 448 & & & 613 & & & 168 & & & 63 & & & 1292 & & \\
\hline \multicolumn{16}{|c|}{ Contexte du milieu familial } \\
\hline $\begin{array}{l}\text { Adulte non-fumeur } \\
\text { avec enfant }\end{array}$ & 282 & 29,7 & 24,8 & 370 & 30,9 & 26,6 & 108 & 30,2 & 26,6 & 29 & 22,1 & 17,4 & 789 & 29,9 & 25,6 \\
\hline $\begin{array}{l}\text { Adulte non-fumeur } \\
\text { sans enfant }\end{array}$ & 293 & 30,9 & 44,1 & 342 & 28,5 & 42,0 & 114 & 31,8 & 43,0 & 42 & 32,1 & 44,3 & 791 & 30,0 & 42,9 \\
\hline $\begin{array}{l}\text { Adulte fumeur } \\
\text { avec enfant }\end{array}$ & 166 & 17,5 & 10,7 & 243 & 20,3 & 12,8 & 60 & 16,8 & 9,7 & 34 & 25,9 & 17,0 & 503 & 19,1 & 11,8 \\
\hline $\begin{array}{l}\text { Adulte fumeur } \\
\text { sans enfant }\end{array}$ & 207 & 21,8 & 20,4 & 243 & 20,3 & 18,6 & 76 & 21,2 & 20,6 & 26 & 26,9 & 21,3 & 552 & 21,0 & 19,6 \\
\hline Total & 948 & & & 1198 & & & 358 & & & 131 & & & 2635 & & \\
\hline \multicolumn{16}{|l|}{ Revenu familial } \\
\hline$>95000$ & 63 & 8,6 & 10,5 & 144 & 15,7 & 19,8 & 30 & 11,1 & 12,3 & 10 & 9,7 & 11,8 & 247 & 12,2 & 15,2 \\
\hline $55000-95000$ & 158 & 21,3 & 22,8 & 256 & 28,0 & 27,7 & 79 & 29,4 & 31,4 & 16 & 15,5 & 16,8 & 509 & 25,1 & 26,2 \\
\hline$\leq 55000$ & 519 & 70,1 & 66,7 & 516 & 56,3 & 52,5 & 160 & 59,5 & 56,3 & 77 & 74,8 & 71,4 & 1272 & 62,7 & 58,6 \\
\hline Total & 740 & & & 916 & & & 269 & & & 103 & & & 2028 & & \\
\hline \multicolumn{16}{|c|}{ Le total peut différer de la taille de l'échantillon (2648) du fait de valeurs manquantes ou inappropriées. } \\
\hline \multicolumn{16}{|c|}{ b n représente la taille réelle de l'échantillon. } \\
\hline c \% représente les propo & ons non & ondérée & & & & & & & & & & & & & \\
\hline
\end{tabular}

variables. Ainsi, ni l'âge, ni le sexe, ni le statut tabagique du répondant n'ont un impact sur la présence d'une PMSF ; il n’y a pas de différence de PMSF non plus, selon que le répondant vit seul ou non. L'effet de la perception de la nocivité de la FTE sur la santé comme un problème de santé très important sur la PMSF est dans la limite de la significativité. Par contre, le niveau de scolarité demeure influent sur la présence d'une PMSF avec un rapport de prévalences (RP) ajusté de 3,26 et un intervalle de confiance (IC) à $95 \%$ de 2,0 à 5,3 .
L'impact de chacune des caractéristiques du ménage sur l'existence d'une PMSF reste constant que l'on tienne compte ou non des autres variables. La plus influente d'entre elles est la présence d'un adulte non-fumeur qui augmente de 4 fois la probabilité de bannir la fumée de tabac à domicile à celle d'un ménage dans lequel tous les adultes fument. La présence d'un enfant influence peu la décision d'adopter une PMSF. En fait, l'impact de cette caractéristique varie avec l'âge : la présence d'un enfant de moins de 6 ans double significativement la probabilité de prendre des mesures restrictives vis-à-vis de la fumée de tabac tandis que celle d'un préadolescent ou d'un adolescent n'a plus un impact significatif. En ce qui concerne l'existence d'une PMSF, l'effet combiné de la présence d'un enfant lorsqu'un adulte non-fumeur est déjà présent est 7 fois supérieur à celui d'un ménage ne comprenant que l'une ou l'autre de ces personnes.

\section{Comparaison entre le Québec et les autres provinces}

Le tableau 4 présente les variations, selon la province de l'effet ajusté, de chacune des caractéristiques sociodémographiques sur l'existence d'une PMSF. L'effet de 
TABLEAU 3

Rapports de prévalences bruts et ajustés de la politique maison sans fumée (PMSF) des ménages du Québec selon les variables socioéconomiques

\begin{tabular}{|c|c|c|c|c|}
\hline Variables & $\mathbf{n}^{\mathrm{a}}$ & PMSF $(\% P)^{b}$ & RP bruts & RP ajustés ${ }^{c}$ \\
\hline \multicolumn{5}{|l|}{ Variables liées au répondant } \\
\hline \multicolumn{5}{|l|}{ Groupe d'âge (en années) } \\
\hline $18-24$ & 156 & 29,5 & $2,4(1,2-5,0)$ & $0,71(0,3-1,8)$ \\
\hline $25-64$ & 718 & 18,1 & $1,3(0,7-2,5)$ & $0,65(0,3-1,4)$ \\
\hline$\geq 65$ & 69 & 13,0 & 1,0 & 1,0 \\
\hline \multicolumn{5}{|l|}{ Sexe } \\
\hline Femme & 549 & 21,1 & $1,24(0,9-1,7)$ & $1,38(0,9-1,9)$ \\
\hline Homme & 400 & 17,7 & 1,0 & 1,0 \\
\hline \multicolumn{5}{|l|}{ Statut tabagique } \\
\hline Non-fumeur & 162 & 29,6 & $2,45(1,6-3,7)$ & $1,09(0,7-1,7)$ \\
\hline Ancien fumeur & 138 & 31,9 & $2,73(1,8-4,1)$ & $1,46(0,9-2,4)$ \\
\hline Fumeur actuel & 649 & 14,6 & 1,0 & 1,0 \\
\hline \multicolumn{5}{|l|}{ État civil } \\
\hline Conjoint & 560 & 19,8 & $1,76(1,0-3,0)$ & $0,82(0,4-1,5)$ \\
\hline Célibataire & 241 & 24,1 & $2,24(1,3-4,0)$ & $1,21(0,6-2,5)$ \\
\hline Séparé/divorcé & 142 & 11,3 & 1,0 & 1,0 \\
\hline \multicolumn{5}{|l|}{ Niveau de scolarité } \\
\hline Baccalauréat ou plus & 134 & 31,0 & $3,5(2,2-5,5)$ & $3,26(2,0-5,3)$ \\
\hline Postsecondaire & 319 & 28,2 & $3,1(2,1-4,5)$ & $2,73(1,8-4,1)$ \\
\hline Secondaire ou moins & 438 & 11,0 & 1,0 & 1,0 \\
\hline \multicolumn{5}{|l|}{ Perception de la FTE comme } \\
\hline Problème de santé très important & 566 & 23,3 & $1,8(1,3-2,6)$ & $1,46(1,0-2,1)$ \\
\hline $\begin{array}{l}\text { Problème de santé peu } \\
\text { ou pas important }\end{array}$ & 380 & 14,2 & 1,0 & 1,0 \\
\hline
\end{tabular}

Variables liées au ménage

Présence de $\geq 1$ adulte non-fumeur

\begin{tabular}{lrrrr} 
Oui & 576 & 27,6 & $4,70(3,1-7,2)$ & $3,81(2,3-6,4)$ \\
Non & 373 & 7,5 & 1,0 & 1,0 \\
\hline Présence d'enfants & & & & \\
Oui & 449 & 21,8 & $1,29(0,9-1,8)$ & $1,32(0,9-1,9)$ \\
Non & 500 & 17,8 & 1,0 & 1,0 \\
\hline
\end{tabular}

Âge de l'enfant (en années)

\begin{tabular}{|c|c|c|c|c|}
\hline$\leq 5$ & 155 & 27,7 & $1,79(1,2-2,7)$ & $1,9(1,1-3,1)$ \\
\hline $6-17$ & 293 & 18,8 & $1,08(0,7-1,6)$ & $1,1(0,7-1,6)$ \\
\hline Absence d'enfant & 500 & 20,7 & 1,0 & 1,0 \\
\hline \multicolumn{5}{|l|}{ Effet du contexte familial } \\
\hline Adulte non-fumeur avec enfant & 283 & 28,6 & $7,1(3,7-13,8)$ & $6,5(3,0-13,9)$ \\
\hline Adulte non-fumeur sans enfant & 293 & 26,6 & $6,5(3,3-12,5)$ & $5,6(2,7-11,6)$ \\
\hline Adulte fumeur avec enfant & 166 & 10,2 & $2,0(0,9-4,5)$ & $2,4(1,0-5,5)$ \\
\hline Adulte fumeur sans enfant & 207 & 5,3 & 1,0 & 1,0 \\
\hline
\end{tabular}

a Le total peut différer de 949 du fait de valeurs manquantes.

b Les proportions de la PMSF sont pondérées.

c Les rapports de prévalences sont ajustés pour les autres variables. certaines variables s'observe unanimement dans les 4 provinces bien que le degré d'influence varie. Ainsi, la présence d'un adulte non-fumeur dans le ménage est la caractéristique la plus fortement associée à l'existence d'une PMSF; elle augmente de près de 4 fois la probabilité de bannir le tabac à domicile au Québec et en NouvelleÉcosse alors qu'en Colombie-Britannique, cette probabilité est seulement doublée. La seconde variable associée à la PMSF est la présence d'un enfant âgé de moins de 6 ans; on note cependant une association plus faible et non significative au Québec tandis qu'en Nouvelle-Écosse et en Ontario elle triple significativement la PMSF. En revanche, l'effet de la présence d'un enfant de plus de 6 ans ne s'observe plus qu'en Colombie-Britannique où l'on constate un impact remarquablement constant quelque soit le groupe d'âge de l'enfant.

Les autres caractéristiques ont une influence qui varie selon la province. La présence d'un enfant quelque soit son âge a un effet sur l'existence d'une PMSF en Ontario et en Colombie-Britannique; bien que non significative, la force de cette association en Nouvelle-Écosse est proche de 2. La probabilité d'une existence de PMSF est 4 fois plus élevée chez les adultes de 18 à 24 ans et 25 à 64 ans que chez ceux de plus de 65 ans en Colombie-Britannique. L'influence du statut tabagique s'observe en Ontario, les anciens fumeurs étant significativement plus prompts à adopter une PMSF que les non-fumeurs et les fumeurs actuels. Le niveau de scolarité du répondant est significativement associé à la prise de mesures restrictives à domicile au Québec et en Ontario. Ni le sexe, ni le fait de vivre ou non avec un conjoint n'influencent la présence d'une PMSF dans aucune des provinces retenues.

\section{Discussion}

La présence d'un adulte non-fumeur dans un ménage comprenant au moins un fumeur est le facteur principal relié à l'existence d'une PMSF, aussi bien au Québec que dans les 3 autres provinces qui ont fait l'objet de cette étude. Ceci confirme les résultats de Borland à Victoria, Australie ${ }^{10}$ selon lesquels un «fumeur vivant avec des 
TABLEAU 4

Variations des effets des caractéristiques sociodémographiques sur l'adoption de la PMSF selon la province

\begin{tabular}{|c|c|c|c|c|}
\hline Variables & $\begin{array}{c}\text { Québec } \\
\text { RPa (IC à 95\%) }\end{array}$ & $\begin{array}{c}\text { Ontario } \\
\text { RP (IC à 95\%) }\end{array}$ & $\begin{array}{l}\text { Colomnie-Br. } \\
\text { RP (IC à 95\%) }\end{array}$ & $\begin{array}{l}\text { Nouvelle-Écosse } \\
\text { RP (IC à 95\%) }\end{array}$ \\
\hline \multicolumn{5}{|l|}{ Variables liées au répondant } \\
\hline \multicolumn{5}{|l|}{ Groupe d'âge } \\
\hline $18-24$ & $0,7(0,4-1,2)$ & $1,86(1,09-3,2)$ & $3,6(1,5-8,6)$ & $1,0(0,4-2,4)$ \\
\hline $25-64$ & $0,5(0,3-0,8)$ & $1,42(0,91-2,2)$ & $3,5(1,7-7,2)$ & $1,3(0,3-5,1)$ \\
\hline$\geq 65$ & 1,0 & 1,0 & 1,0 & 1,0 \\
\hline \multicolumn{5}{|l|}{ Sexe } \\
\hline Femme & $1,3(0,9-1,8)$ & $1,3(1,0-1,6)$ & $1,1(0,7-1,7)$ & $0,8(0,4-1,7)$ \\
\hline Homme & 1,0 & 1,0 & 1,0 & 1,0 \\
\hline \multicolumn{5}{|l|}{ Statut tabagique } \\
\hline Non-fumeur & $1,2(0,8-1,8)$ & $1,2(0,8-1,6)$ & $0,5(0,3-0,9)$ & $0,5(0,1-1,5)$ \\
\hline Ancien fumeur & $1,5(0,9-2,3)$ & $1,6(1,1-2,3)$ & $0,5(0,2-0,9)$ & $1,5(0,5-4,8)$ \\
\hline Fumeur actuel & 1,0 & 1,0 & 1,0 & 1,0 \\
\hline \multicolumn{5}{|l|}{ État civil } \\
\hline Conjoint & $0,6(0,4-1,1)$ & $1,0(0,7-1,4)$ & $0,8(0,4-1,4)$ & $0,9(0,3-2,3)$ \\
\hline Célibataire & $1,0(0,5-1,7)$ & $1,0(0,6-1,5)$ & $0,9(0,5-1,8)$ & $0,9(0,3-2,8)$ \\
\hline Séparé & 1,0 & 1,0 & 1,0 & 1,0 \\
\hline \multicolumn{5}{|l|}{ Niveau de scolarité } \\
\hline Baccalauréat ou plus & $2,8(1,7-4,5)$ & $2,4(1,7-3,4)$ & $1,6(0,9-2,9)$ & $1,5(0,5-4,6)$ \\
\hline Postsecondaire & $2,4(1,6-3,4)$ & $1,5(1,1-2,0)$ & $2,1(1,2-3,5)$ & $2,2(0,5-5,7)$ \\
\hline Secondaire ou moins & 1,0 & 1,0 & 1,0 & 1,0 \\
\hline \multicolumn{5}{|l|}{ Perception de la FTE comme } \\
\hline Problème de santé très important & $1,5(1,0-2,1)$ & $1,3(0,9-1,7)$ & $1,5(0,9-2,5)$ & $1,6(0,6-4,1)$ \\
\hline $\begin{array}{l}\text { Problème de santé peu } \\
\text { ou pas important }\end{array}$ & 1,0 & 1,0 & 1,0 & 1,0 \\
\hline \multicolumn{5}{|l|}{ Variables liées au ménage } \\
\hline \multicolumn{5}{|l|}{ Présence de $\geq 1$ adulte non-fumeur } \\
\hline Oui & $3,8(2,4-5,9)$ & $3,5(2,6-4,7)$ & $2,4(1,5-3,9)$ & $3,7(1,6-8,8)$ \\
\hline Non & 1,0 & 1,0 & 1,0 & 1,0 \\
\hline \multicolumn{5}{|l|}{ Présence d'enfant } \\
\hline Oui & $1,2(0,9-1,8)$ & $1,8(1,4-2,3)$ & $2,6(1,6-4,1)$ & $1,9(0,9-4,3)$ \\
\hline Non & 1,0 & 1,0 & 1,0 & 1,0 \\
\hline \multicolumn{5}{|l|}{ Âge de l'enfant (en années) } \\
\hline$\leq 5$ & $1,6(1,0-2,6)$ & $3,0(2,1-4,2)$ & $2,5(1,4-4,7)$ & $3,5(1,2-10,5)$ \\
\hline 6-17 & $1,01(0,7-1,5)$ & $1,3(0,9-1,8)$ & $2,7(1,6-4,6)$ & $1,4(0,6-3,5)$ \\
\hline Absence d'enfant & 1,0 & 1,0 & 1,0 & 1,0 \\
\hline \multicolumn{5}{|l|}{ Effet de la pression familiale } \\
\hline Adulte non-fumeur avec enfant & $5,7(2,8-11,3)$ & $6,6(4,3-10,2)$ & $6,3(3,1-12,5)$ & $7,9(1,9-32,8)$ \\
\hline Adulte non-fumeur sans enfant & $5,3(2,7-10,5)$ & $4,1(2,6-6,3)$ & $2,4(1,2-4,7)$ & $4,1(1,0-16,3)$ \\
\hline Adulte fumeur avec enfant & $2,0(0,9-4,5)$ & $2,2(1,4-3,5)$ & $2,5(1,2-5,3)$ & $2,2(0,5-9,6)$ \\
\hline Adulte fumeur sans enfant & 1,0 & 1,0 & 1,0 & 1,0 \\
\hline
\end{tabular}

a Les rapports de prévalence sont ajustés pour les autres variables. non-fumeurs, a 4.7 fois (IC à $95 \%=3.7$ à 6.0) plus de probabilité de fumer hors du domicile qu'un fumeur cohabitant avec seulement des fumeurs ". L'influence de cette variable est cependant moindre en Colombie-Britannique, suggérant que le fumeur est assez sensibilisé à la nocivité de la FTE pour adopter de lui-même une PMSF, ou il est plus porté à fumer dehors; la Colombie-Britannique a un climat plus doux que les autres provinces inclus dans cette étude. L'intérêt de ce facteur, dans le cadre des interventions sur la promotion de la santé, est de suggérer une réflexion sur la mise au point d'une campagne plus orientée vers les non-fumeurs vivant avec les fumeurs. Ainsi, contrairement aux campagnes classiques dirigées vers le fumeur pour l'exhorter à fumer hors du domicile, une campagne incitant le nonfumeur à faire preuve de moins de tolérance vis-à-vis de la fumée de tabac à domicile pourrait contribuer à réduire l'exposition à la FTE.

Étonnamment, la présence dans le ménage fumeur québécois d'un enfant, tous âges confondus, n'est pas lié à l'existence d'une PMSF contrairement à ce qui s'observe en Colombie-Britannique et en Ontario. Cependant, cette attitude s'améliore lorsque l'enfant a moins de 6 ans. Bien que l'influence de la présence d'un enfant dans le ménage puisse paraître faible sur l'existence d'une PMSF au Québec, il faut signaler que ceci est un progrès par rapport aux résultats d'une étude menée par Statistiques Canada en 1996 selon laquelle ni la présence d'un enfant, ni son âge n'affectaient le fait de fumer à la maison ${ }^{7}$. Néanmoins, ces données sont peu encourageantes du fait de l'influence positive de la PMSF sur une moindre prévalence du tabagisme à l'adolescence. En effet, certains auteurs dont Farkas ${ }^{11}$ estime qu'un adolescent habitant dans un ménage sans fumée a 0.74 fois la probabilité de devenir fumeur comparativement à un adolescent vivant dans une maison sans restriction. De plus, il observe que les adolescents déjà fumeurs ont deux fois plus de chance de devenir des ex-fumeurs lorsqu'ils vivent dans un domicile sans fumée. Wakefield ${ }^{12}$ a observé que la transition au cours des différentes étapes de l'accoutumance au tabac est significativement ralentie quand 
l'adolescent rapporte que son domicile a adopté une PMSF. Les résultats de cette étude suggèrent d'importantes implications opérationnelles pour les programmes de santé publique puisqu'ils laissent supposer que les parents sont plus enclins à adopter une PMSF lorsque leurs enfants sont en bas âge mais le deviennent moins lorsque ceux-ci sont plus âgés. Outre le bénéfice de l'adoption d'une PMSF pour la santé de l'enfant, peut-être faut-il mettre de l'avant le bénéfice sur le comportement tabagique ultérieur de l'enfant.

La perception de la FTE comme un problème de santé très important a un effet dans les limites de la significativité. La portion des répondants percevant la FTE comme un problème de santé peu ou pas important en 2001 est substantielle (40\%); bien que cette proportion ait pu diminué depuis la collecte des données, il s'agit d'une variable d'un grand intérêt en santé publique puisque modifiable.

Contrairement aux résultats de certains auteurs, l'existence d'une PMSF dans un ménage n'est pas liée au sexe du répondant ni au Québec ni dans les 3 autres provinces qui ont fait l'objet de cette étude; ces résultats diffèrent de ceux de Gilpin $^{13}$ et de Merom $^{14}$ qui observent que les femmes adoptent significativement moins souvent la PMSF que les hommes avec respectivement, un rapport de cotes de 0.72 IC $(0.61$ à 0.84$)$ et de 0.84 IC $(0.72$ à 0.96). On observe que le groupe d'âge du répondant n'a pas d'influence sur l'existence d'une PMSF par les ménages québécois. Ceci est surprenant quand on tient compte du fait que les participants de 18 à 24 ans appartiennent à une génération connaissant mieux les méfaits de la FTE et aussi au fait qu'ils sont plus susceptibles d'avoir de jeunes enfants à domicile.

La force de cette étude est d'identifier des facteurs liés à l'adoption d'une PMSF dans les ménages québécois "fumeurs ». En santé publique, il est primordial, lorsque cela est possible, d'identifier des facteurs expliquant une partie d'un problème touchant une population et qui se prêtent à modification. Les résultats de cette étude suggèrent que la FTE à domicile est modifiable, au moins en partie par une moindre tolérance d'un adulte non fumeur cohabitant avec un fumeur et par l'amélioration de la perception de la FTE comme un problème de santé très important. Ils suggèrent également que les parents perçoivent moins le bénéfice de la PMSF lorsque leurs enfants ont 6 ans et plus. Ces hypothèses, une fois confirmées, permettront de modifier les interventions de promotion de la santé. De fait, une étude chinoise ${ }^{15}$ sur les facteurs prédictifs d'une faible PMSF rapporte la présence d'un partenaire fumeur (rapport de cotes $=2,78, p<0,05)$.

L'on pourrait arguer de l'ancienneté des données puisque la collecte remonte à 2001. Cependant, les résultats de cette étude demeurent tout à fait pertinents pour plusieurs raisons. D'abord, il faut bien convenir que c'est le seul indice épidémiologique dont nous disposons puisqu'il s'agit de la première étude du genre réalisée sur un échantillon représentatif de toute la population canadienne; ensuite, au meilleur de nos connaissances, aucune étude n'a encore été publiée sur les facteurs associés à la politique maison sans fumée au Québec. De ce fait, les résultats de cette étude peuvent servir de comparaison à des études ultérieures. Enfin, les résultats de l'Enquête de surveillance de l'usage du tabac au Canada (ESUTC) de 2006 montrent que ces données sont toujours d'actualité puisque le Québec demeure encore au-dessus de la moyenne canadienne pour ce qui est de l'exposition des enfants âgés de 0 à 17 ans à la fumée de tabac secondaire en milieu privé; ainsi, $18.4 \%$ des 0 à 11 ans et $25.8 \%$ des 11 à 17 ans y sont régulièrement exposés par comparaison, respectivement, à 3 et $7 \%$ en Colombie-Britannique, 5 et $8.9 \%$ en Ontario, et 10.5 et $18 \%$ en Nouvelle-Écosse ${ }^{8}$.

La Loi sur le tabac du Québec interdisant de fumer dans divers endroits (locaux de soins de santé, garderies, services sociaux; écoles et établissements supérieurs, locaux de sports en salle, d'activités culturelles ou de loisirs et transports en commun) existe avant 2001. Depuis le 31 mai 2006, il existe une interdiction de fumer dans les bars et restaurants. L'impact de cette nouvelle loi sur la diminution de l'acceptabilité sociale du tabac est certes cumulatif à la Loi sur le tabac mais elle ne nous paraît pas de nature à bouleverser la PMSF au point d'invalider les résultats de la présente étude.

Cette étude comporte quelques limites qu'il convient de souligner. D'abord le taux de réponse de $62 \%$ est modeste et pourrait inciter à réduire la confiance que l'on a dans le signalement de la PMSF. Cependant, il s'inscrit dans la fourchette des taux couramment obtenus avec la méthode utilisée. En effet, comparativement aux décennies 1970, 1980, 1990, l’on constate une baisse tendancielle des taux de réponse aux enquêtes ${ }^{16-17}$.

Ensuite, la catégorisation de la variable PMSF en deux niveaux, PMSF quotidienne et PMSF occasionnelle ou jamais, tend à regrouper dans la dernière catégorie aussi bien des domiciles n'ayant jamais une PMSF que des domiciles dans lesquels la règle est occasionnellement enfreinte. Cependant, bien que la catégorisation en trois groupes soit plus proche de la réalité, notre intérêt se porte vers les domiciles où le statut de PMSF est quotidien afin d'identifier les variables expliquant un tel comportement; en outre la faiblesse des effectifs de certains sous groupes limite le choix de cette manière de procéder.

En ce qui concerne la pondération de l'estimation des prévalences dans cette étude, les coefficients auraient dû être calculés à partir de la distribution des ménages selon les statistiques nationales. En l'absence de telles statistiques, ces coefficients ont été obtenus à partir de la distribution des ménages participant à l'enquête, laquelle constitue une bonne estimation des données nationales. Par conséquent les prévalences estimées dans notre étude sont probablement proches de la réalité.

Enfin, les résultats de la NouvelleÉcosse devraient être interprétés avec prudence compte tenu de la faible taille de l'échantillon $(n=132)$. Ce contexte rend difficile la distinction entre une absence d'association et l'incapacité de l'étude à détecter une association si elle existe. 


\section{Conclusion}

La performance de la stratégie de lutte contre le tabagisme au Québec, la baisse de l'acceptabilité sociale vis-à-vis du tabagisme et les lois restrictives dans les lieux publics et de travail sont à l'origine de l'ampleur de la réduction de la prévalence du tabagisme. Cependant les enfants québécois ne profitent pas encore tous de cette évolution et demeurent plus exposés à la FTE que les enfants des autres régions du Canada. Les résultats de la présente étude permettent de préciser le profil des enfants exposés à la FTE en milieu privé. Ils suggèrent, en effet, que les enfants vivant dans un ménage fumeur qui ne comprend aucun adulte non-fumeur constituent un groupe plus à risque d'exposition à la FTE que ceux qui vivent avec au moins un adulte non-fumeur. Ils laissent également supposer que l'adoption d'une PMSF est acceptée pour les enfants en bas âge mais l'est moins pour les enfants plus âgés, ce qui pourrait avoir d'importantes implications opérationnelles pour les programmes de santé publique. Compte tenu de l'influence considérable de la présence d'un adulte non-fumeur dans le cadre de l'existence d'une PMSF, une autre implication s'impose sur les interventions de promotion de la santé, et pourrait concerner la mise au point de campagne visant les non-fumeurs vivant avec des fumeurs afin de les inciter à faire preuve de moins de tolérance vis-àvis de la fumée de tabac à domicile.

\section{Références}

1. Centre National de Documentation sur le Tabac et la Santé. Faits saillants, La fumée de tabac dans l'environnement : ses conséquences générales pour la santé. Ottawa, Conseil canadien pour le contrôle du tabac, 1996.

2. Glantz SA, Parmley WW. Passive smoking and heart disease. Mechanisms and risk. JAMA. 1995 Apr 5; 273(13):1047-53.

3. Sandler DP, Wilcox AJ, Everson R.B. Cumulative effects of lifetime passive smoking on cancer risk. Lancet. 1985 Feb 9; 1 (8424):312-5.
4. Pizacani BA, Martin DP, Stark MJ et al. Household smoking bans: which households have them and do they work? Prev Med. 2003; Jan; 36(1):99-107.

5. Ashley MJ, Cohen J, Ferrence $\mathrm{R}$ et al. Smoking in the home: changing attitudes and current practices. Am J Public Health. 1998 May; 88(5):797-800.

6. Ekos Research Associates Inc. As Assessment of Knowledge, Attitudes and Practices Concerning Environmental Tobacco Smoke. Rapport final. Ottawa (Ontario), 1995, 31 mars. Soumis à Santé Canada.

7. Ashley MJ, Ferrence R. Environmental tobacco smoke (ETS) in home environments: a discussion paper prepared for Health Canada's Strategic Planning Workshop to Reduce ETS, Ottawa (Ontario), The Ontario Tobacco Research Unit, 1995, 19-20 octobre.

8. Santé Canada. Enquête de surveillance de l'usage du tabac au Canada. Section sur les foyers, Ottawa, Santé Canada, 2006. Disponible à : http://www.hc-sc.gc.ca/ hl-vs/tobac-tabac/research-recherche/

stat/_ctums-esutc_2006/ann-table9_f.html

9. Northrup DA. Environmental tobacco smoke in the home: a national survey technical documentation, Toronto, Institute for Social Research, York University, 2002.

10. Borland R, Mullins R, Trotter L et al. Trends in environmental tobacco smoke restrictions in the home in Victoria, Australia. Tob Control. 1999; 8(3):266-71.

11. Farkas AJ, Gilpin EA, White MM et al. Association between household and workplace smoking restrictions and adolescent smoking. JAMA. 2000; 284(6):717-22.

12. Wakefield MA, Chaloupka FJ, Kaufman NJ et al. Effect of restrictions on smoking at home, at school, and in public places on teenage smoking: cross sectional study. BMJ. 2000; 321(7257):333-7.
13. Gilpin EA, White MM, Farkas AJ et al. Home smoking restrictions: which smokers have them and how they are associated with smoking behavior. Nicotine Tob Res. 1999; 1(2):153-62.

14. Merom D, Rissel C. Factors associated with smoke-free homes in NSW: results from the 1998 NSW Health Survey. Aust N Z J Public Health. 2001; 25(4):339-45.

15. Mak YW, Loke AY, Abdullah AS, Lam TH. Household smoking practices of parents with young children, and predictors of poor household smoking practices. Public Health. 2008 Nov;122(11):1199-209. Epub 2008 Jul 10.

16. Dunkelberg WC, Day GS. Non response bias and call backs in sample surveys. J Mark Res. 1973;10:160-8.

17. Dillman DA. Mail and internet surveys: the tailored design method. New York, John Wiley \& Sons, 2000. 464p. 